relationship to the development of shoulder pain in this group of swimmers, perhaps further investigation of strength ratios in future large prospective investigations into risk factors in the development of shoulder pain is warranted.

https://doi.org/10.1016/j.jsams.2017.09.230

40

Developing a nitrate, nitrite, and nitrosamine food and beverage composition database for use with a nitrate food frequency questionnaire: A systematic review

N. McMahon ${ }^{1, *}$, T. Pavey ${ }^{2}$, B. Desbrow ${ }^{3}$, M. Leveritt $^{1}$

${ }^{1}$ School of Human Movement and Nutrition Sciences, University of Queensland, Australia

${ }^{2}$ School of Exercise and Nutrition Sciences, Queensland University of Technology, Australia

${ }^{3}$ School of Allied Health Sciences, Griffith University, Australia

Introduction: There is convincing evidence to demonstrate that dietary nitrate $\left(\mathrm{NO}_{3}{ }^{-}\right)$supplementation (beetroot juice or sodium nitrate capsules) improves exercise performance, but there is a lack of research on the potential ergogenic benefits of $\mathrm{NO}_{3}{ }^{-}$intake through nitrate-rich foods. The substantial variability in dietary $\mathrm{NO}_{3}{ }^{-}$consumption and the $\mathrm{NO}_{3}{ }^{-}$concentrations in food highlights the need for a validated dietary assessment tool to accurately determine $\mathrm{NO}_{3}{ }^{-}$and nitrite $\left(\mathrm{NO}_{2}{ }^{-}\right)$intake. The purpose of this systematic review was to create a comprehensive database that includes the concentrations of $\mathrm{NO}_{3}{ }^{-}, \mathrm{NO}_{2}{ }^{-}$, and nitrosamines in foods and beverages. To be used in conjunction with a newly developed shortterm food frequency questionnaire (FFQ), this database will provide quantitative data for use in epidemiological studies examining dietdisease relationships or sports performance research.

Methods: Relevant databases such as Agricola, Cochrane Library, Embase, Medline, PubMed, Scopus and Web of Science were searched for the following search terms 'nitrate OR nitrite OR nitrosamine AND content AND food AND nutrient AND composition' from 1960 to September 2016. Food composition quantitative values relating to $\mathrm{NO}_{3}{ }^{-}, \mathrm{NO}_{2}{ }^{-}$, and nitrosamines were extracted, as well as publication year and country, food, and analytical information. The literature search identified 7,573 studies, of which 274 met the inclusion criteria.

Results: After data collation, the database contained a total of $3498 \mathrm{NO}_{3}{ }^{-}, 2134 \mathrm{NO}_{2}{ }^{-}$, and 954 nitrosamines individual food and beverage estimates from sixty different countries. To account for the potential impact of environmental influences on $\mathrm{NO}_{3}{ }^{-}$values (such as temperature, atmospheric humidity, amount of sunlight exposure), where multiple reference sources were available for single food or beverage items, foods were coded based on year and country of analysis. By presenting the data in this user-friendly format, the user can select values based on their own specific requirements.

Discussion: This database has potential implications for quantification of dietary intakes of $\mathrm{NO}_{3}{ }^{-}, \mathrm{NO}_{2}{ }^{-}$, and nitrosamines in both public health and clinical settings. Use of this database in future interventions may guide the development of future research and help lead to dietary nitrate supplementation recommendations around optimal dosing strategies, which population is most likely to benefit, and under which conditions dietary nitrates are likely to be most effective for enhancing performance. $\mathrm{NO}_{3}{ }^{-}$.
41

Assuming rater reliability of a movement competency screen - Is it true?

K. Mann ${ }^{1, *}$, N. O’Dwyer ${ }^{2}$, S. Bird ${ }^{3}$, S. Edwards ${ }^{4}$

${ }^{1}$ Charles Sturt University, Australia

${ }^{2}$ University of Sydney, Australia

3 James Cook University, Australia

${ }^{4}$ University of Newcastle, Australia

Introduction: Due to the substantial financial and social benefits of reducing injury prevalence, movement competency screen (MCS) is an extremely common tool utilised across all levels of the sporting community. A key requirement of a MCS is that it must be reliable, and although previous research has claimed various MCS demonstrate good rater reliability, there are major limitations in this previous research methodology including low sample size and inappropriate statistical methods. This study therefore aims to determine the reliability of a field-based MCS in novice and expert raters, and determine if there is a learning effect present for both rater and participant.

Methods: Fifty-five pre-elite youth athletes with no previous MCS experience were videoed performing the MCS on three separate occasions (session 1 , session 2 and session 3 ), with minimum 4 week washout between sessions. Twelve raters watch and rated each participants MCS three times in a randomised order with minimum 4 week washout between each rating. Raters either viewed three sessions comprising of either only session 1 only ( $n=3$ expert; $n=6$ novice) to assess rater reliability or session 1,2 and 3 ( $n=3$ expert; $n=6$ novice) to assess if learning effect for participants. Kappa, intra-class correlation and percentage agreement were calculated for each of the six movements and the total MCS score to determine intra- and inter-rater reliability.

Results: Total MSC score when viewing the same video showed excellent reliability, with multiple sessions reducing from excellent to moderate reliability. When viewing the same video on each occasion, reliability across individual movement tasks displayed poor reliability for both novice and expert raters for both Kappa score and percentage agreement. Nevertheless, when combined to a total score, excellent reliability for both expert and novice raters in ICC scores were exhibited.

Discussion: Unexpectantly, both novice and experts were too variable to achieve reliable rating of individual movement tasks when viewing the same video repeatedly, yet the total MSC score displayed excellent reliability. This difference in reliability suggests that although individual MSC score cannot be reliably used, the total MSC score can be used reliably to classify movement competency. This study is the first to demonstrate an athlete learning effect whereby total MSC score reliability increased when an athlete performed MSC on another occasion, highlighting that an individual must be familiarised with movements to ensure that the screening is valid.

https://doi.org/10.1016/j.jsams.2017.09.232

42

Changes in salivary stress hormone levels associated with competition in male competitive swimmers

\section{T. Matsumto}

Kokushikan University, Japan

Introduction: It is known that salivary cortisol and testosterone levels, which are reported to reflect the stress response, are cor- 